\title{
Disseminating research findings using a massive online open course for maximising impact and developing recommendations for practice
}

Nancy Preston ${ }^{1 *}$, Jeroen Hasselaar ${ }^{2}$, Sean Hughes ${ }^{1}$, Alex Kaley ${ }^{3}$, Lisa Linge-Dahl${ }^{4}$, Idiko Radvanyi ${ }^{5}$, Phil Tubman $^{3}$, Karen Van Beek ${ }^{6}$, Sandra Varey ${ }^{1}$ and Sheila Payne ${ }^{1}$

\begin{abstract}
Background: Developing recommendations for how we deliver healthcare is often left to leading experts in a field. Findings from the Integrated Palliative Care in cancer and chronic conditions (InSup-C) study, which aimed to identify best practice in integrated palliative care in cancer, chronic obstructive pulmonary disease (COPD) and heart failure, led to recommendations developed through an expert consultation process. We also wanted to develop these recommendations further with participants who were largely clinicians and members of the public.

Methods: Results from the InSup- $C$ study were disseminated through a three-week massive open online course (MOOC) which ran in 2016, 2017 and 2019. The first course helped develop the final recommendations, which were ranked by MOOC participants in the subsequent courses. MOOC participants were predominantly clinicians, but also academics and members of the public. They rated how important each recommendation was on a 9 point scale (9 most important). Descriptive statistics were used to analyse the ratings. The results were compared to findings from the consultation.

Results: Five hundred fifteen completed the last part of the course where the recommendations were ranked, of which 195 (38\%) completed the ratings. The top recommendations related to: need to expand palliative care to non-malignant conditions; palliative care needs to include different dimensions of care including physical, psychological and spiritual; policies and regulations assessments should be made regularly; palliative care integration should be mandatory; and there should be greater availability of medicines. These differed compared to the top ranked recommendations by the consultation panel in relation to the importance of leadership and policy making. This may indicate that clinicians are more focused on daily care rather than the (inter) national agenda.

* Correspondence: n.j.preston@lancaster.ac.uk

${ }^{1}$ International Observatory on end of Life Care, Lancaster University, Lancaster, UK

Full list of author information is available at the end of the article

(c) The Author(s). 2020 Open Access This article is licensed under a Creative Commons Attribution 4.0 International License, which permits use, sharing, adaptation, distribution and reproduction in any medium or format, as long as you give appropriate credit to the original author(s) and the source, provide a link to the Creative Commons licence, and indicate if changes were made. The images or other third party material in this article are included in the article's Creative Commons licence, unless indicated otherwise in a credit line to the material. If material is not included in the article's Creative Commons licence and your intended use is not permitted by statutory regulation or exceeds the permitted use, you will need to obtain permission directly from the copyright holder. To view a copy of this licence, visit http://creativecommons.org/licenses/by/4.0/ The Creative Commons Public Domain Dedication waiver (http://creativecommons.org/publicdomain/zero/1.0/) applies to the data made available in this article, unless otherwise stated in a credit line to the data. 


\begin{abstract}
(Continued from previous page)
Conclusions: Whilst both sets of recommendations are important, our study shows that we need to include the views of clinicians and the public rather than rely upon leading expert opinion alone. To keep recommendations fresh we need both the input of clinicians, the public and experts. When disseminating findings, MOOCs offer a useful way to gain greater reach with clinicians and the public, and importantly could be a vehicle to validate recommendations made by leading expert panels.
\end{abstract}

Keywords: Integrated, Palliative care, E-learning, Recommendations

\section{Background}

In 2014 the World Health Assembly passed a resolution urging governments to integrate palliative care into existing health care services [1]. However, it is unclear which are the best models to achieve this [2-4]. A variety of guidelines are available but these have largely been generated through consensus meetings with a limited number of experts, although some have included results of systematic literature reviews and consensus methods [5-7].

The authors were part of a Framework Programme 7 (FP7) European Commission funded study, called InSup$\mathrm{C}$, which was designed to evaluate best practice in integrating palliative care services in five countries: Netherlands, Belgium, Germany, Hungary and the United Kingdom (UK) [8-10]. Using multiple embedded case study methods, it aimed to find out the best ways to deliver care to people who have advanced cancer, heart failure or lung disease as they come towards the end of their lives. We included 23 integrated palliative care initiatives across the five countries to identify factors associated with good practice in integrating palliative care services.

We presented our findings to a group of international experts and key stakeholders from Europe in 2016. This consensus group then prepared some recommendations based upon consensus methods [11]. However, very few clinicians were involved and those that were, were in senior roles. Hence, we wanted to assess the value of previously generated recommendations by gaining the perspectives of a greater number of people involved in palliative care but especially clinicians.

\section{Integration of palliative care may occur at three levels}

Macro - incorporation of palliative care into national health care strategies and resource allocation plans;

Meso - inclusion of palliative care into regional, local and organisational health care services;

Micro - working at the level of specific patients and families, ensuring that palliative care operates in association with other medical disciplines such as oncology, neurology and geriatrics so that patients experience seamless care [12].

\section{Methods}

This paper seeks to examine the congruence between participant's opinions enrolled on a MOOC about key recommendations for the further integration of palliative care at three levels: micro, a meso and a macro.

\section{Design of the study}

As part of the dissemination of the InSup-C project we ran a free online course called Palliative Care Making it Work [8]. This Massive Open Online Course (MOOC) ran over 3 weeks and included various activities for the participants including an introduction to Palliative Care, how to integrate it and exemplars of best practice.

\section{Effective practice}

In the final week of the MOOC, participants were invited to take part in a questionnaire survey which was developed for this study (see Supplementary file), where participants were presented with recommendations from the InSup-C project, and invited to rate each item in terms of its importance. The survey took approximately 15-20 min and could be completed by anyone taking part in the MOOC. Taking part in the independent survey was entirely optional and MOOC participants were not incentivised to do so, either through financial incentives or additional course credits.

The Integrated Palliative Care MOOC was delivered on three separate occasions (October 2016, June 2017 and June 2018). The MOOC 1 (2016) independent survey presented participants with 11 recommendations and was taken directly from the findings of the InSup-C project. MOOCs 2 \& $3(2017 / 18)$ were delivered following a consultation workshop attended by international policy makers, including national and international experts in palliative care, in September 2016. The independent survey for MOOCs $2 \& 3$ therefore presented participants with an additional 15 recommendations, integrating the MOOC 1 survey recommendations and findings from the consultation workshop. This is in keeping with the Delphi method which advocates an iterative process for building consensus and congregating expert opinion [13, 14].

As part of the independent survey, participants were invited to rate the importance of each recommendation 
item using a Likert scale of 0-9 (where 0 indicated lowest and 9 indicating highest priority). Open comments on the items were possible.

\section{Sample and settings}

The integrated palliative care MOOC was made available to the general public through the Futurelearn platform [15]. Futurelearn is a private company, which offers a diverse selection of courses from leading universities and cultural institutions from around the world. The MOOC was promoted internationally via the Futurelearn platform, and was completed by participants from 148 countries worldwide. No eligibility restrictions were placed on enrolment, and the course was intended for anyone with an interest in palliative care.

A total of 9682 people enrolled on the Integrated Palliative Care MOOC called 'Palliative Care: Making it work', which was delivered on three separate occasions (October 2016, June 2017 and June 2018). The recommendations survey was one of the last steps of the 3 week course. As with many MOOCs only about small proportion (10-20\%) of those who enrol completed the MOOC [16]. For MOOC 1 (6489 enrolled), 794 people reached the recommendations stage of the MOOC and 460 completed the survey (58\%). These results, together with the results from the consensus work shop [11], formed the definitive set of recommendations for MOOC 2 and 3. For MOOCs 2 and 3 (3193 enrolled) a total number of 515 people reached the part of the course containing the recommendations survey. One hundred ninety-five completed the survey (representing a $38 \%$ response rate).

In this paper we report on findings from the independent recommendations survey conducted for MOOCs 2 and $3(2017 / 18)$ as these two surveys asked participants to rank an identical set of recommendations and therefore offer comparable data for analysis.

\section{Demographic details of participants}

When students enrolled on the MOOC, they were invited to complete a short set of questions regarding their sociodemographic background (e.g. gender, age, highest qualification and occupation). This was optional and MOOC participants were not obliged to give this information in order to complete the course. Of the 1293 people who accessed the independent survey, around $22 \%$ of those provided responses to the demographic questionnaire. While we cannot, therefore, report on the demographic characteristics for the entire survey sample, by matching the data of those participants who accessed the independent survey with those who completed the demographic questionnaire, we are able to make some general observations about the participants, based on the information given. The majority of participants were women (around 87\%) and people in mid to later life appear to represent the dominant age groups $(68 \%)$. The most commonly reported profession was health and social care (73\%) with the majority of participants (83\%) educated to degree level or above. Just over half of respondents (54\%) were from the United Kingdom, most closely followed by other predominantly English speaking countries, including Australia (8\%) Canada (5\%) and the US (3\%). However, we are unable to analyse the data according to these variables.

\section{Data collection and analysis}

We report descriptive statistics for the survey items. For each item, we report mean agreement to determine the highest ranked items and standard deviation (SD) to determine the degree of consensus. Respondents made very few narrative comments, but these helped to clarify recommendations.

The data from the independent survey reported in this paper were undertaken as part of the MOOC online course and were consultative in nature. As such, we did not seek formal research ethics approval, as this was not required for this activity, and is congruent with other Transparent expert consultation (TEC) studies [17]. Participants were informed that their data would be anonymised and stored according to Lancaster University's data protection policy and in accordance with general data protection regulations (GDPR), prior to commencement of the survey.

\section{Results}

The recommendations were attributed to three levels.

- macro - national/international level

- meso - organisational/institutional level

- micro - interactions between patients, families and social care professionals

\section{Ranking of recommendations}

Following the survey, analysis of the degree of consensus on all recommendations showing mean and SD are displayed in Tables 1, 2 and 3, across Macro, Meso and Micro levels. Whilst MOOC participants gave high priority to all of the recommendations listed in the independent survey (typically a score of between 7 and 9) our analysis of the degree of consensus did enable us to rank the recommendations in order of importance, relative to one another and the top 5 recommendations are listed in Table 4.

Overall, all recommendations indicated high or medium level consensus on their importance [11]. Two recommendations (RECs) (RECs 3 and 6 - macro level) were very similar and ranked highest so are treated as one which related to palliative care services needing to 
Table 1 Recommendations for Integrated Palliative Care at Macro Level

\begin{tabular}{|c|c|c|c|c|}
\hline No. & MACRO Recommendations & Mean & SD & $\begin{array}{l}\text { Overall } \\
\text { Ranking }\end{array}$ \\
\hline 6 & $\begin{array}{l}\text { National palliative care regulations and policies should be extended to apply to all patients with palliative care } \\
\text { needs, not just those with cancer. }\end{array}$ & 8.59 & 0.94 & 1 \\
\hline 3 & $\begin{array}{l}\text { Palliative care regulations and policies should be extended to apply to non-cancer patients as well (for example } \\
\text { COPD, heart failure and dementia). }\end{array}$ & 8.52 & 1.11 & 3 \\
\hline 13 & $\begin{array}{l}\text { Palliative care should be integrated into mandatory education for undergraduate medical, health and social care } \\
\text { professionals. }\end{array}$ & 8.42 & 1.21 & 5 \\
\hline 14 & $\begin{array}{l}\text { Continuing professional development for all health and social care professionals should include coverage of } \\
\text { integrated palliative care. }\end{array}$ & 8.26 & 1.07 & 9 \\
\hline 5 & $\begin{array}{l}\text { For integration to work, new and creative ways of securing resources and specific funding should be established } \\
\text { which can support the palliative care infrastructure. }\end{array}$ & 7.95 & 1.28 & 18 \\
\hline 10 & There needs to be national level strategic lobbying to develop and fund better integrated palliative care. & 7.95 & 1.39 & 19 \\
\hline 16 & Disease/condition specific national policies should integrate palliative care. & 7.95 & 1.30 & 20 \\
\hline 17 & There is a need for strong leadership to advocate for integrated palliative care. & 7.94 & 1.51 & 21 \\
\hline 18 & There is a need to invest in the development of future integrated palliative care leadership skills. & 7.88 & 1.44 & 23 \\
\hline 15 & Social care should be part of integrated palliative care. & 7.51 & 1.22 & 26 \\
\hline
\end{tabular}

be broadened to beyond cancer. The next highest recommendation was about ensuring palliative care encompassed all dimensions of care including physical, spiritual and psychological (REC 25 - micro level). The third highest related to the need for regular assessment (REC 26 - micro level). The fourth was the need for palliative care to be mandated into undergraduate education of health care professionals (REC 13 - macro level). The fifth highest was about making essential medicines affordable and available (REC 22 - micro level). There was a greater diversity in responses to a number of macro level statements no. 5, 10, 16, 17, 18 and two Micro level statements no.7 and 20, as indicated by larger SDs. This suggests that there was less agreement regarding the importance of these recommendations.

\section{Policies need to extend palliative care beyond cancer (RECS 3 and 6)}

Members of the MOOC came from 148 countries and, for some, the idea of extending palliative care beyond cancer was novel. This may be why introducing the idea of extending palliative care beyond cancer was rated so highly. Even for those who already recognised this need, they may have felt in day-to-day practice services were still predominantly aimed at patients with cancer hence they felt the need to reinforce this recommendation.

\section{Including different dimensions of care including physical, spiritual and psychological}

Physical care has become increasingly predominant in palliative care and perhaps the MOOC raised the profile of non-physical care with participants recognising that this also needed prioritising. This was endorsed by using the WHO definition of palliative care in the MOOC which may have been new to some of the participants who included generalists as well as specialists in palliative care.

\section{Need for regular assessments shared with the care team} For the clinicians in the MOOC, regular assessment may have been prioritised to maintain good daily care on a

Table 2 Recommendations for Integrated Palliative care at Meso Level

\begin{tabular}{|c|c|c|c|c|}
\hline No. & MESO Recommendations & Mean & SD & $\begin{array}{l}\text { Overall } \\
\text { Ranking }\end{array}$ \\
\hline 4 & $\begin{array}{l}\text { The digital transfer of information should be integrated within and across different palliative care services and general } \\
\text { services including community and hospital teams, and patients and families. }\end{array}$ & 8.36 & 1.09 & 7 \\
\hline 2 & $\begin{array}{l}\text { An information hub, (online or a face-to-face central resource for the coordination of information exchange), with a care } \\
\text { co-ordination team should be established to contribute to the integration of palliative care services across the area. }\end{array}$ & 8.12 & 1.28 & 12 \\
\hline 21 & Raise awareness of integrated palliative care for senior managers and policy makers. & 8.08 & 1.26 & 13 \\
\hline 1 & Outcome measures to assess quality of integrated palliative care services should be developed. & 8.05 & 1.17 & 14 \\
\hline 23 & Outcomes of integrated palliative care should be audited and benchmarked. & 8.05 & 1.25 & 15 \\
\hline 12 & Develop alliances within and between health care sectors to build better integration. & 8.01 & 1.21 & 17 \\
\hline
\end{tabular}


Table 3 Recommendations for Integrated Palliative Care at Micro level

\begin{tabular}{|c|c|c|c|c|}
\hline No. & MICRO Recommendations & Mean & SD & $\begin{array}{l}\text { Overall } \\
\text { Ranking }\end{array}$ \\
\hline 25 & $\begin{array}{l}\text { Integrated palliative care should encompass different dimensions of care including physical, psychological and } \\
\text { spiritual aspects. }\end{array}$ & 8.58 & 0.95 & 2 \\
\hline 26 & $\begin{array}{l}\text { Integrated palliative care should involve assessments which are regularly updated and shared with other healthcare } \\
\text { professionals within the care team. }\end{array}$ & 8.45 & 0.97 & 4 \\
\hline 22 & Access to readily available and affordable essential medicines are necessary for integrated palliative care. & 8.42 & 1.20 & 6 \\
\hline 9 & $\begin{array}{l}\text { Develop systems that provide adequate out-of-hours integrated palliative care so that health care practitioners can } \\
\text { maintain their work/life balance. }\end{array}$ & 8.30 & 1.21 & 8 \\
\hline 24 & Raise public awareness about palliative care and its integration with healthcare. & 8.24 & 1.13 & 10 \\
\hline 19 & Establish needs based referral systems to guide timely referrals to integrated palliative care. & 8.16 & 1.17 & 11 \\
\hline 8 & $\begin{array}{l}\text { Clinical protocols should be introduced to ensure integration of palliative care services for patients and families } \\
\text { regardless of the setting where they are treated. }\end{array}$ & 8.04 & 1.22 & 16 \\
\hline 20 & Establish a single point of contact for integrated palliative care at local level. & 7.91 & 1.58 & 22 \\
\hline 7 & $\begin{array}{l}\text { Building of informal relationships between health professionals are a foundation for formal structures which are } \\
\text { pivotal for the integration of palliative care. }\end{array}$ & 7.81 & 1.43 & 24 \\
\hline
\end{tabular}

micro-level. The need for regular assessment was not highlighted in the MOOC but gaining greater involvement with the multidisciplinary team was. Sharing results of assessments may have been linked to the importance of working in multi-disciplinary contexts.

\section{Education}

Education was considered a key priority by MOOC participants. For them, palliative care should be integrated into mandatory education for undergraduate medical, health and social care professionals (REC 13) (ranked 5 th in order of importance). Also regarded as important (although lower ranked) was the inclusion of integrated palliative care in the continuing professional development of health and social care professionals (REC 14). This suggests that MOOC participants regarded education about integrated palliative care to be a higher priority for those at the beginning of their health and social care careers.

\section{Access to and affordability of essential medications}

Education about access to opioids world-wide was covered extensively in the MOOC and may have been novel, particularly for the UK participants who made up about
$50 \%$ of the cohort. Equally they may have been reflecting on the timely provision of medicines when they may be required at short notice as a patient's condition deteriorates.

\section{Discussion}

The ranking of the recommendations about how to integrate palliative care are broadly similar to those produced through the consensus meeting [11]. However the MOOC participants who were largely clinicians prioritised the need for education. This may be because they recognised their own needs whereas the members of the consensus group were already highly informed about the need for palliative care and were focussing on how to develop palliative care at a national or international level $[18,19]$. If clinicians recognise that they need more education about how to integrate palliative care, then this need should be rated higher with policy makers, such as those at the consensus meeting, as without policy makers' support change will not happen. The members of consensus group highlighted a greater need to raise public awareness [20], which was a lesser concern for the MOOC participants indicating that they were perhaps more focussed on the need to change day to day

Table 4 Top 5 Recommendations across all levels (MACRO, MESO and MICRO)

\begin{tabular}{|c|c|c|c|}
\hline No. & Recommendation & Mean & SD \\
\hline 6 & $\begin{array}{l}\text { National palliative care regulations and policies should be extended to apply to all patients with palliative care needs, not just } \\
\text { those with cancer/ Palliative care regulations and policies should be extended to apply to non-cancer patients as well (for ex- } \\
\text { ample COPD, heart failure and dementia). }\end{array}$ & $\begin{array}{l}8.59 / \\
8.52\end{array}$ & $\begin{array}{l}0.94 / \\
1.11\end{array}$ \\
\hline 25 & Integrated palliative care should encompass different dimensions of care including physical, psychological and spiritual aspects. & 8.58 & 0.95 \\
\hline 26 & $\begin{array}{l}\text { Integrated palliative care should involve assessments which are regularly updated and shared with other healthcare professionals } \\
\text { within the care team. }\end{array}$ & 8.45 & 0.97 \\
\hline 13 & Palliative care should be integrated into mandatory education for undergraduate medical, health and social care professionals. & 8.42 & 1.21 \\
\hline 22 & Access to readily available and affordable essential medicines are necessary for integrated palliative care. & 8.42 & 1.2 \\
\hline
\end{tabular}


care, rather greater changes across society. Another area of difference was in relation to leadership. Participants from the consensus group, who could be argued are already in leadership roles, rated the need higher (REC 17, 18) than MOOC participants. Palliative care has evolved as a largely social movement with greater focus on developing relationships as a means to developing palliative care [10]. The emphasis has been on charismatic champions, rather than other types of leadership, so those conducting day to day care will be operating more on this relational level than seeing leadership as a necessary priority.

MOOC participants gave less priority in general to policy recommendations. One way to interpret this is may be that how participants view issues relating to policy is influenced by the level at which they operate. For example, if you operate at a more strategic or national level then policy making would be perceived as having a greater significance than it would for service or 'ground level' practitioners. This was similar to recommendations about audit and outcome measures where the consensus group gave this higher priority. Evidence and audit are needed to influence policy and allocation of resources [21] whereas relationships are potentially more important to influence change in daily practice [10]. Even though MOOC participants recognised the need to establish these informal relationships, (REC 6) they placed greater emphasis on developing alliances within and between health care sectors (REC 11). This was similar to the consensus group, with both groups recognising the need for multiple agency involvement to deliver effective integrated palliative care.

There was a higher priority in relation to the need for integrated palliative care to encompass the different dimensions of palliative care namely physical, psychological and spiritual aspects [1]. This was echoed in some of the feedback from MOOC participants during the course, which demonstrated these were new ideas for some, as many were not specialist palliative care practitioners. This reminds us that palliative care occurs outside of specialist care contexts and more effective involvement and work with generalists providing palliative care is required if change is to be achieved. The MOOC, which included people from 148 countries and from a large range of disciplines, was therefore a useful mechanism to engage people in developing recommendations.

There is a great need for access to good out of hours palliative care [22] services, but this was recognised more by the MOOC participants than by the members of the consensus group. Once again, this may reflect the daily experience of staff. From our own research on integrated palliative care $[9,10]$, we found that in some countries, this was only achieved by practitioners giving personal contact numbers and being permanently 'on call'. Whilst this was not true in all countries, it may go some way to explaining why the MOOC participants rated maintaining a work life balance (REC 9) higher than those at the consensus workshop.

The contrast between the day-to-day needs of practitioners compared to the ratings from the consensus group, was evident in relation to accessibility and affordability of medicines (REC 22), which was rated higher by MOOC participants. This may also be reflective of the experiences of MOOC participants, drawn from around the world and the wide variability in access to medicines. The accessibility and affordability of medicines was included as teaching topic on the MOOC. This may have been one of the first times MOOC participants, particularly those from the UK with a readily available supply of opiates, had realised access was not universal. This contrasts to the consensus group who rated changes at national level through lobbying to develop and gain better funding for integrated palliative care (REC 10) or develop new innovative ways to secure funding (REC 5) as higher. This suggests that MOOC participants concerns around funding are micro-level rather than macro-level related, in contrast to consensus group of international experts.

\section{Study strengths and limitations}

This is the first independent survey that specifically elicits practitioners' (and other professionals') views on integrated palliative care, and compares this large sample with the views of experts [9]. Our study is innovative in that it incorporated a wide range of views on recommendations and priorities for integrated palliative care rather than just the views of experts. Further, all participants were well informed having engaged in a course about integrated palliative care. However, they may not have been representative of all palliative care workers as they had self-selected to attend the course. Even though all participants (MOOC and experts) had similar information about the outcomes of the primary study, their own experiences appeared to influence how they ranked the recommendations. A limitation of the study was that we did not have full demographic information for each participant, although we were able to make some general observations about the characteristics of the participants, based on the socio-demographic information given at the point of enrolment. However, we weren't able to analyse specific variables such as country of origin, role, or gender. Also, as the recommendations came at the end of the course it is unclear if participants ranked items in a way to 'please' the course tutors by ranking certain topics higher which they perceived were presented as important on the course [23]. However this potential limitation was mitigated because the ranking was anonymous. 


\section{Conclusions}

Our findings suggest that although recommendations were similar for MOOC participants and those generated at the consensus workshop, there were some important differences. The MOOC gave a platform to gain a broader range of opinions, including those of clinicians, which is useful when making recommendations for advancing palliative care policy and practice. The MOOC is a useful mechanism for gaining greater dissemination of research but also as a vehicle for producing recommendations.

\section{Supplementary information}

Supplementary information accompanies this paper at https://doi.org/10. 1186/s12904-020-00564-7.

Additional file 1. Questionnaire developed for this study

\section{Abbreviations}

InSuP-C: Integrated Palliative Care in cancer and chronic conditions; COPD: Chronic obstructive pulmonary disease; MOOC: Massive open online course; FP7: Framework Programme 7; UK: United Kingdom; SD: Standard deviation; TEC: Transparent expert consultation; GDPR: General data protection regulations; RECs: Recommendations

\begin{abstract}
Acknowledgements
The authors are grateful to all the participants who came on the MOOC and took time to complete the recommendations. InSup-C was coordinated by Radboud University Medical Center, Nijmegen, The Netherlands. Other partners are: University Hospital, Bonn, Germany; International Observatory on End-of-Life Care, Lancaster University, Lancaster and Sheffield University, United Kingdom; University of Pecs Medical School, Pecs, Hungary; University Hospital Leuven, Leuven, Belgium; World Health Organization, Geneva, Switzerland; European Association for Palliative Care (EAPC OnLus), Milan, Italy; Erasmus University, Rotterdam, The Netherlands; University of Navarra, Navarra, Spain; Mount Sinai Medical Center, New York, USA
\end{abstract}

\section{Authors' contributions}

NP, SH, SV, JH, PT, LLD, KvB, IR and SP developed and ran the MOOC. NP, SP, $\mathrm{JH}$ and $\mathrm{SH}$ designed the study and planned the analysis. NP led the writing of the paper. NP, SP and AK participated in the analysis. JH supervised the overall study. All authors read and approved the final manuscript.

\section{Funding}

The research leading to these results has received funding from the European Union's Seventh Framework Programme (FP7/2007-2013) under grant agreement $n^{\circ} 335555$. The funders had no influence on the data collection, analysis or interpretation of the data.

\section{Availability of data and materials}

All data are archived at Lancaster University and may be obtained from the first author.

\section{Ethics approval and consent to participate}

This paper reports on the dissemination activities linked to the InSuP-C study where NHS ethical approval was obtained on 27 March 2014 from NRES Committee North West - Lancaster (Ref: 14/NW/0140). No ethical requirements were required to conduct the recommendations survey. Responses to the online survey indicated implied consent and participant were told that results would be used to inform recommendations to be published. This was in line with the Futurelearn research policies and terms and conditions explained to participants on the MOOC.

\section{Consent for publication}

Not applicable.

\section{Competing interests}

SP, AK, SV and JH have declared no potential conflicts of interest with respect to the research, authorship, and/or publication of this article. Nancy Preston is a section editor for BMC Palliative Care.

\section{Author details}

${ }^{1}$ International Observatory on end of Life Care, Lancaster University, Lancaster, UK. ²Department of Anaesthesiology, Pain and Palliative Medicine, Radboud University Medical Center, Nijmegen, The Netherlands. ${ }^{3}$ Lancaster University, Lancaster, UK. ${ }^{4}$ Department of Palliative Care, University Hospital Bonn, Bonn, Germany. ${ }^{5}$ University of Pécs Medical School, Pécs, Hungary. ${ }^{6}$ Department of Radiation-Oncology and Palliative Medicine, University Hospital Gasthuisberg, Leuven, Belgium.

Received: 12 August 2019 Accepted: 17 April 2020

Published online: 22 April 2020

\section{References}

1. Organization WH. Strengthening of palliative care as a component of integrated treatment throughout the life course. J Pain Palliat Care Pharmacother. 2014;28:130-4.

2. Siouta N, Van Beek K, Van der Eerden M, et al. Integrated palliative care in Europe: a qualitative systematic literature review of empirically-tested models in cancer and chronic disease. BMC Palliat Care. 2016:15:56.

3. Fusi TOA, Froggatt F, Preston N. Palliative care provision for patients with advanced chronic obstructive pulmonary disease: a systematic integrative literature review (under review). COPD: J Chron Obstruct Pulmon Dis. 2018; 15(6):600-11.

4. Kaasa $\mathrm{S}$, Loge JH, Aapro M, et al. Integration of oncology and palliative care: a lancet oncology commission. Lancet Oncol. 2018;19(11):e588-653.

5. Siouta N, van Beek K, Preston N, et al. Towards integration of palliative care in patients with chronic heart failure and chronic obstructive pulmonary disease: a systematic literature review of European guidelines and pathways. BMC Palliat Care. 2016;15:18.

6. Van Beek K, Siouta N, Preston N, et al. To what degree is palliative care integrated in guidelines and pathways for adult cancer patients in Europe: a systematic literature review. BMC Palliat Care. 2016;15:26.

7. Siouta N, Van Beek K, Payne S, et al. Is the content of guidelines/pathways a barrier for the integration of palliative Care in Chronic Heart Failure (CHF) and chronic pulmonary obstructive disease (COPD)? A comparison with the case of cancer in Europe. BMC Palliat Care. 2017;16:62.

8. van der Eerden M, Csikos A, Busa C, et al. Experiences of patients, family and professional caregivers with Integrated Palliative Care in Europe: protocol for an international, multicenter, prospective, mixed method study. BMC Palliat care. 2014;13:52.

9. den Herder-van der Eerden M, Hasselaar J, Payne $\mathrm{S}$, et al. How continuity of care is experienced within the context of integrated palliative care: a qualitative study with patients and family caregivers in five European countries. Palliat Med. 2017;31:946-55.

10. den Herder-van der Eerden M, van Wijngaarden J, Payne S, et al. Integrated palliative care is about professional networking rather than standardisation of care: a qualitative study with healthcare professionals in 19 integrated palliative care initiatives in five European countries. Palliat Med. 2018;32: 1091-102.

11. Payne S, Hughes S, Wilkinson J, et al. Recommendations on priorities for integrated palliative care: transparent expert consultation with international leaders for the InSuP-C project. BMC Palliat Care. 2019;18:32.

12. SP JH. Integrated palliative care. Nijmegan: Radboud University Medical Centre; 2016.

13. Morita T, Bito S, Kurihara Y, et al. Development of a clinical guideline for palliative sedation therapy using the Delphi method. J Palliat Med. 2005;8: 716-29.

14. Stevinson C, Preston N, Todd C, et al. Defining priorities in prognostication research: results of a consensus workshop. Palliat Med. 2010;24:462-8.

15. Palliative Care: Making it Work, https://www.futurelearn.com/courses/ palliative. 2018, Accessed 12/7/2019 2019

16. Rieber LP. Participation patterns in a massive open online course (MOOC) about statistics. Br J Educ Technol. 2017;48:1295-304.

17. Higginson IJ, Evans CJ, Grande G, et al. Evaluating complex interventions in end of life care: the MORECare statement on good practice generated by a 
synthesis of transparent expert consultations and systematic reviews. BMC Med. 2013;11:111.

18. Schiessl C, Walshe M, Wildfeuer $S$, et al. Undergraduate curricula in palliative medicine: a systematic analysis based on the palliative education assessment tool. J Palliat Med. 2013;16:20-30.

19. Carrasco JM, Lynch TJ, Garralda E, et al. Palliative care medical education in European universities: a descriptive study and numerical scoring system proposal for assessing educational development. J Pain Symptom Manage. 2015;50:516-23 e512.

20. Stjernswärd J, Foley KM, Ferris FD. The public health strategy for palliative care. J Pain Symptom Manag. 2007;33:486-93.

21. Sleeman $K E$, de Brito $M$, Etkind $S$, et al. The escalating global burden of serious health-related suffering: projections to 2060 by world regions, age groups, and health conditions. Lancet Glob Health. 2019;7:e883-92.

22. Fordham FT, McMillan S, Best S, et al. How current UK research addresses out of hours palliative care BMJ Supportive \& Palliative Care. 2016;6:384.

23. Grimm P. Social desirability bias. Wiley international encyclopedia of marketing; 2010.

\section{Publisher's Note}

Springer Nature remains neutral with regard to jurisdictional claims in published maps and institutional affiliations.

Ready to submit your research? Choose BMC and benefit from:

- fast, convenient online submission

- thorough peer review by experienced researchers in your field

- rapid publication on acceptance

- support for research data, including large and complex data types

- gold Open Access which fosters wider collaboration and increased citations

- maximum visibility for your research: over $100 \mathrm{M}$ website views per year

At BMC, research is always in progress.

Learn more biomedcentral.com/submissions 